\title{
Progressive Freeze-Concentration of Model Liquid Food
}

\author{
Ling LiU, ${ }^{1}$ Osato MIYAWAKI ${ }^{2}$ and Kozo NaKamura \\ Department of Applied Biological Chemistry, The University of Tokyo, 1-1-1 Yayoi, Bunkyo-ku, Tokyo 113, Japan
}

Received February 19, 1997; Accepted June 13, 1997

\begin{abstract}
Progressive freeze-concentration utilizes the concentration phenomena of a solute at the ice-solution interface moving from one end of a vessel to the other end. It is characterized by having only a single ice crystal in the system so that the separation of the ice crystal from the concentrated solution is very easy compared with the conventional method of freeze-concentration. Progressive freeze-concentration was applied to a solution containing glucose and/or blue dextran as a model liquid food. The freeze-concentration ratio and apparent partition coefficient of a solute between the ice and the solution phases were strongly dependent on the moving speed of the freezing front and the stirring speed at the ice-solution interface. A lower moving speed and a higher stirring speed produced a better freeze-concentration ratio.
\end{abstract}

Keywords: progressive freezing, normal freezing, freeze-concentration, partition constant, freezing front, supercooling

Among the methods of concentration of liquid food, freeze-concentration has several advantages: low energy requirements, low process temperature preventing undesirable chemical and biochemical changes, and minimal loss of flavors and aromas (Kobayashi, 1991; Shirai, 1991; Ramteke et al. 1993). Therefore, freeze-concentration has been applied to concentrations of liquid food such as fruit juices (Deshpande et al., 1982; Bayindirli et al., 1993) and dairy products (Hartel \& Espinel, 1993).

A typical freeze-concentration system is composed of an ice nucleator, a recrystallizer, and an ice crystal separator (Huige \& Thijssen, 1972). The ice nucleator produces small ice crystals which are transferred into the recrystallizer and grown large by Ostwald ripening. The ice crystals are then collected and transferred to the ice crystal separator and washed with water to purify the ice crystals and recover the solutes involved. The key technology in this system is how to grow ice crystals in the recrystallizer large enough to facilitate the separation between the ice crystals and solutes. Therefore, the mechanism of the ice crystal growth in the recrystallizer has been extensively investigated and supercooling and secondary nucleation were identified clear to be major factors preventing ice crystals from growing large (Omran \& King, 1974; Shirai et al., 1985; Hartel \& Chung, 1993).

In the previous paper (Bae et al., 1994), we proposed a progressive freeze-concentration as a method of freezeconcentration of liquid food based on a concept completely different from the conventional method of freeze-concentration described above. In the progressive freezing or normal freezing (Gouw, 1968), freeze-concentration occurs in the freezing process with the ice phase growing from one end of the sample vessel to the other end. In this method, the freeze-concentration proceeds at the moving ice front and

${ }^{1}$ Present address: Food Engineering Department, Zhengzhou Institute of Light Industry, \#5, Dong Feng Road, Zhengzhou Henan, People Repub. China.

${ }^{2}$ To whom correspondence should be addressed. only a single ice crystal is formed so that it is easy to separate the ice crystals from the concentrated solution.

In this paper, glucose and blue dextran in an aqueous model liquid food were concentrated by progressive freezeconcentration. The effects of operating conditions on the freeze-concentration ratio will be discussed.

\section{Theoretical}

In the progressive freeze-concentration, the mechanism of concentration is the exclusion of solute molecules from the moving ice front, the interface between the ice and solution phases. The apparent partition coefficient $(K)$ between the solution and the ice phase can be defined as follows:

$$
K=C_{\mathrm{S}} / C_{\mathrm{L}}
$$

where $C_{\mathrm{S}}[\mathrm{wt} \%]$ and $C_{\mathrm{L}}[\mathrm{wt} \%]$ are solute concentrations in the ice and solution phases, respectively. At the moment when the solution volume is $V_{\mathrm{L}}$ in the process of progressive freeze-concentration, assume a small volume increase $\left(-d V_{\mathrm{L}}\right)$ in the ice phase. The following equation is obtained from the mass balance of the solute by assuming complete mixing in the solution phase and no mixing in the ice phase

$$
C_{\mathrm{L}} V_{\mathrm{L}}=-C_{\mathrm{S}} d V_{\mathrm{L}}+\left(C_{\mathrm{L}}+d C_{\mathrm{L}}\right)\left(V_{\mathrm{L}}+d V_{\mathrm{L}}\right)
$$

Using Eq. (1),

$$
\frac{\left(d C_{\mathrm{L}} / C_{\mathrm{L}}\right)}{\left(d V_{\mathrm{L}} / V_{\mathrm{L}}\right)}=K-1
$$

This equation can be integrated as follows:

$$
(1-K) \log \left(V_{\mathrm{L}} / V_{0}\right)=\log \left(C_{0} / C_{\mathrm{L}}\right)
$$

where $C_{0}[\mathrm{wt} \%]$ is the initial concentration of the solute before freeze-concentration, and $V_{0}$ is the initial volume of the solution to be concentrated.

\section{Materials and Methods}

Materials $\mathrm{D}-(+)$-Glucose was purchased from Nacalai Tesque, (Kyoto). Blue dextran (BD) was obtained from Sigma Chemical (St. Louis). Glucose assay kit (F-kit) was 


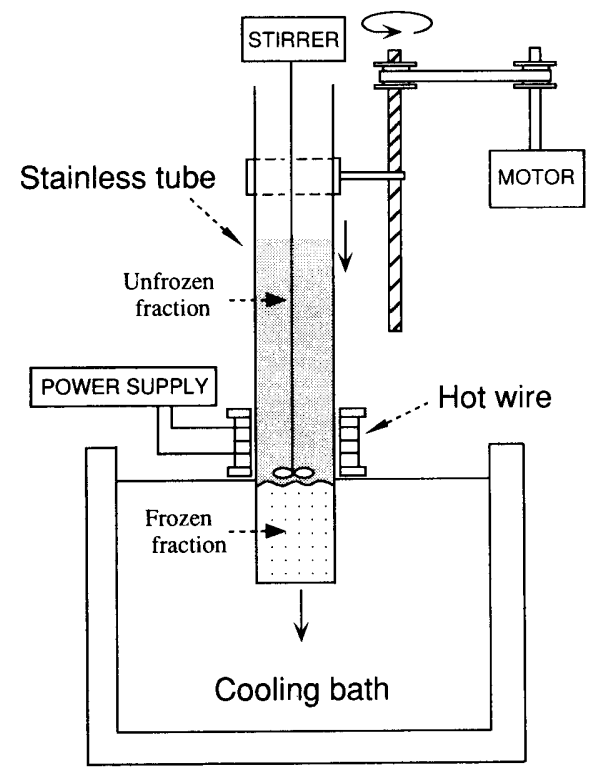

Fig. 1. Apparatus for progressive freeze-concentration.

from Boehringer Mannheim Yamanouchi (Tokyo). Icenucleating protein solution (from Xanthomonas campestris INXC-1) was supplied by Q.P. Corp.

Apparatus and experimental procedure Figure 1 shows the apparatus for progressive freeze-concentration, which is composed of a cylindrical sample vessel of stainless steel $(48 \mathrm{~mm} \phi, 197.5 \mathrm{mmH})$, a cooling bath, and a driving system to move the sample vessel into the cooling bath $\left(-10^{\circ} \mathrm{C}\right)$ at a constant speed $(0.16-2.37 \mathrm{~cm} / \mathrm{h})$. The sample vessel was equipped with a propeller inside to stir the solution at the ice-solution interface and a hot-wire heater outside to control the level of the ice front in the sample vessel.

Glucose (5 wt\%) and/or BD (0.006 wt\%) was dissolved in pure water and poured into the sample vessel to start the freeze-concentration. In sampling, the progressive freezeconcentration process was stopped and the solution and the ice phases were removed for an assay. Glucose and $\mathrm{BD}$ were analyzed spectroscopically (Beckmann DU-65) using the F-kit and directly from the optical density at $256 \mathrm{~nm}$, respectively. For the data at a different sampling time, the whole procedure was repeated again from the start.

When ice-nucleating protein was added, the ice-nucleating protein solution was used as received and added to the sample at $0.01-0.1 \mathrm{wt} \%$.

\section{Results and Discussion}

Figure 2 shows changes in the concentrations of glucose and $\mathrm{BD}$ both in the solution and ice phases during the progressive freeze-concentration of a mixed solution of glucose and BD with a moving speed of the freezing front of $2 \mathrm{~cm} / \mathrm{h}$ and a stirring speed of $600 \mathrm{rpm}$. It is clearly understood that the concentrations of solutes in the ice phase changed little with time and were much lower than those in the solution phase while the concentrations of solutes in the solution phase increased with time showing that the concentration process effectively proceeded.

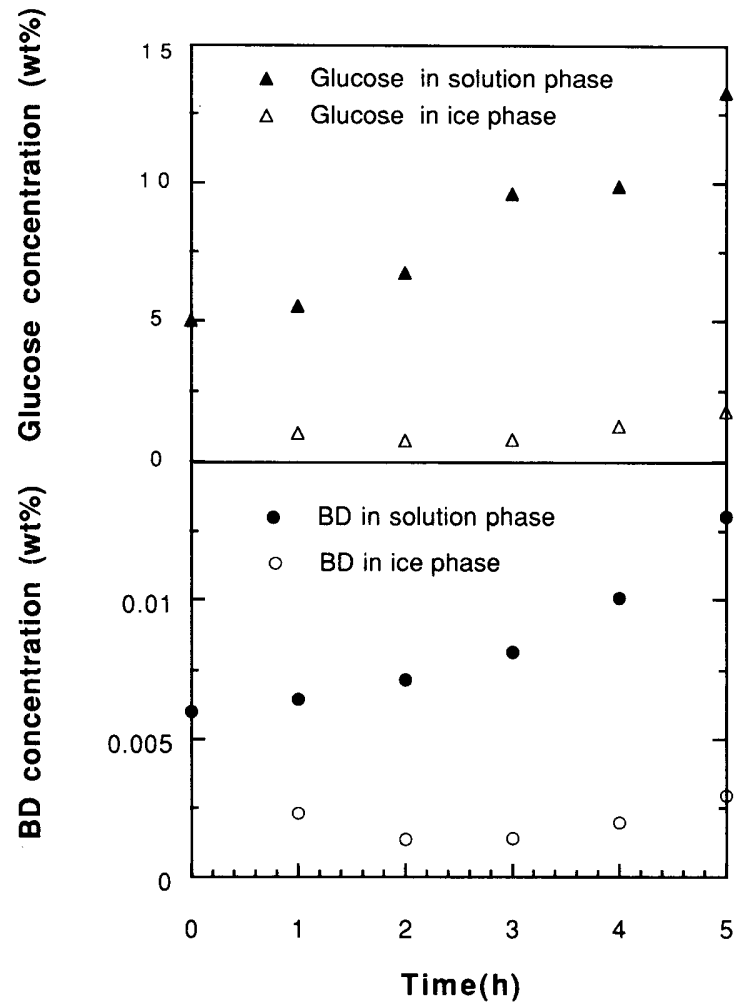

Fig. 2. Changes in concentrations of glucose and BD with time in the solution and the ice phases during progressive freeze-concentration of a mixed solution of glucose and BD (stirring speed, $600 \mathrm{rpm}$; moving speed of the freezing front, $2 \mathrm{~cm} / \mathrm{h}$ ).

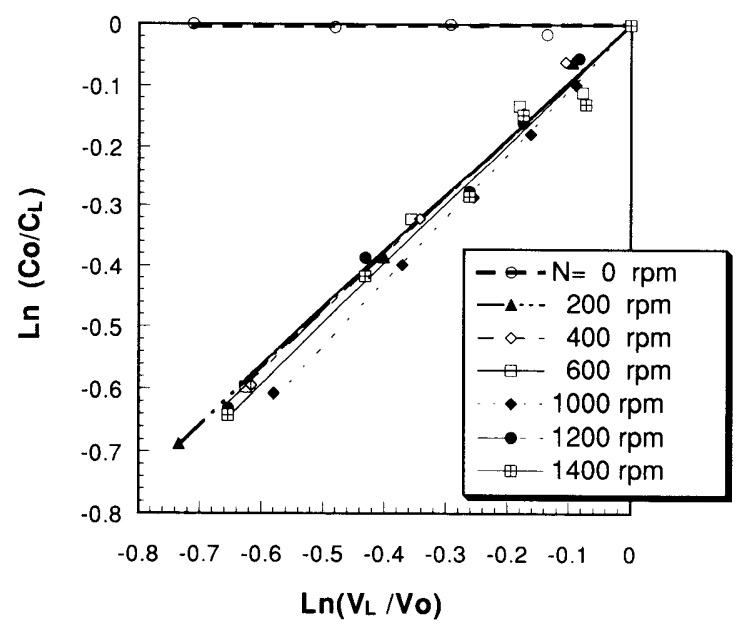

Fig. 3. Relationship between the relative concentration in solution and the volume ratio of the unfrozen phase during progressive freeze-concentation of a BD solution at various stirring speeds. Moving speed of the freezing front was kept at $2 \mathrm{~cm} / \mathrm{h}$.

Figure 3 shows the relationship between the relative concentration $\left(C_{0} / C_{\mathrm{L}}\right)$ of $\mathrm{BD}$ in the solution phase and the volume ratio of the unfrozen phase $\left(V_{\mathrm{L}} / V_{0}\right)$ based on Eq. (4). The linear relationship between the two shows that the hypothesis in Eq. (4) is correct and that the apparent partition constants defined by Eq. (1) can be calculated from the slope 
of the line. No freeze-concentration occurred without stirring ( $K=1$ ), and $K$ decreased drastically with stirring. This shows that the mass transfer near the ice-solution interface is important for effective progressive freeze-concentration.

The effect of stirring on the apparent partition constant is shown in Fig. 4. A higher stirring rate generally produced a higher freeze-concentration ratio although the partition coefficient changed depending on the situation. In the single component solutions, the partition constant was much lower for BD compared with glucose, probably reflecting the difference in the solute concentration in the sample. In the mixed solution of glucose and BD, however, the partition constant was lower for glucose than $\mathrm{BD}$, suggesting some mechanism of discrimination between the two components at the ice-solution interface.

Figure 5 shows the effect of the moving speed of the freezing front on the concentration change in the solute with

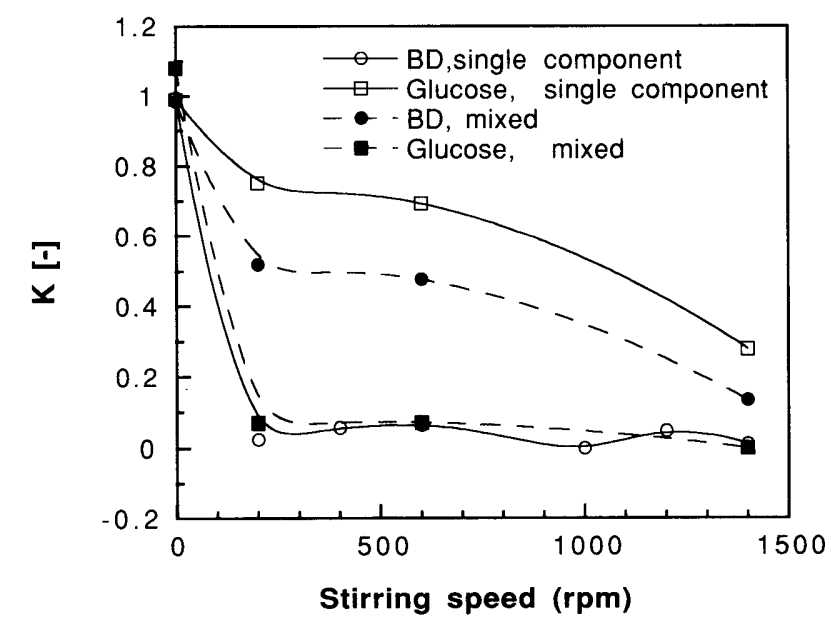

Fig. 4. Apparent partition coefficient and its dependence on stirring speed at the ice-solution interface in progressive freeze-concentration of a mixed solution of glucose and BD. Moving speed of the freezing front was kept at $2 \mathrm{~cm} / \mathrm{h}$. time during the process of progressive freeze-concentration for a mixed system of glucose and BD. A slower moving speed of the ice front produced a better freeze-concentration ratio.

The effect of the moving speed of the freezing front on the apparent partition constant is shown in Fig. 6. A lower moving speed of the freezing front yielded a higher concentration efficiency both for glucose and BD. The dependence of the solute concentration in the ice phase on the moving speed of the freezing front is shown in Fig. 7. A slower moving
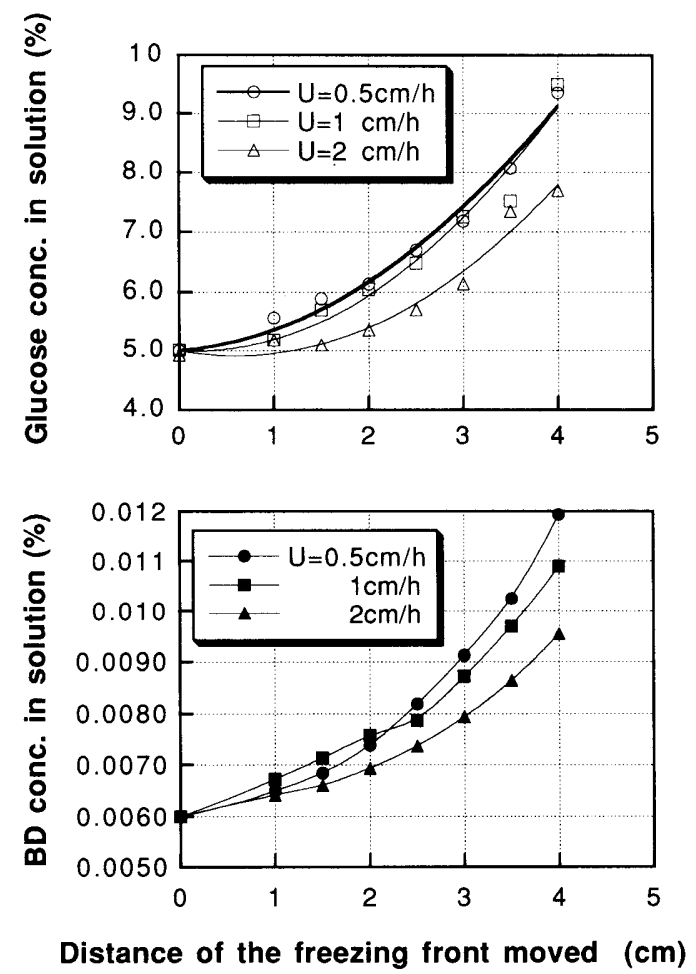

Fig. 5. Effect of moving speed of freezing front on changes in concentrations of glucose and $\mathrm{BD}$ in the solution phase during progressive freeze-concentration of a mixed solution of glucose and BD at a stirring speed of $1400 \mathrm{rpm}$.
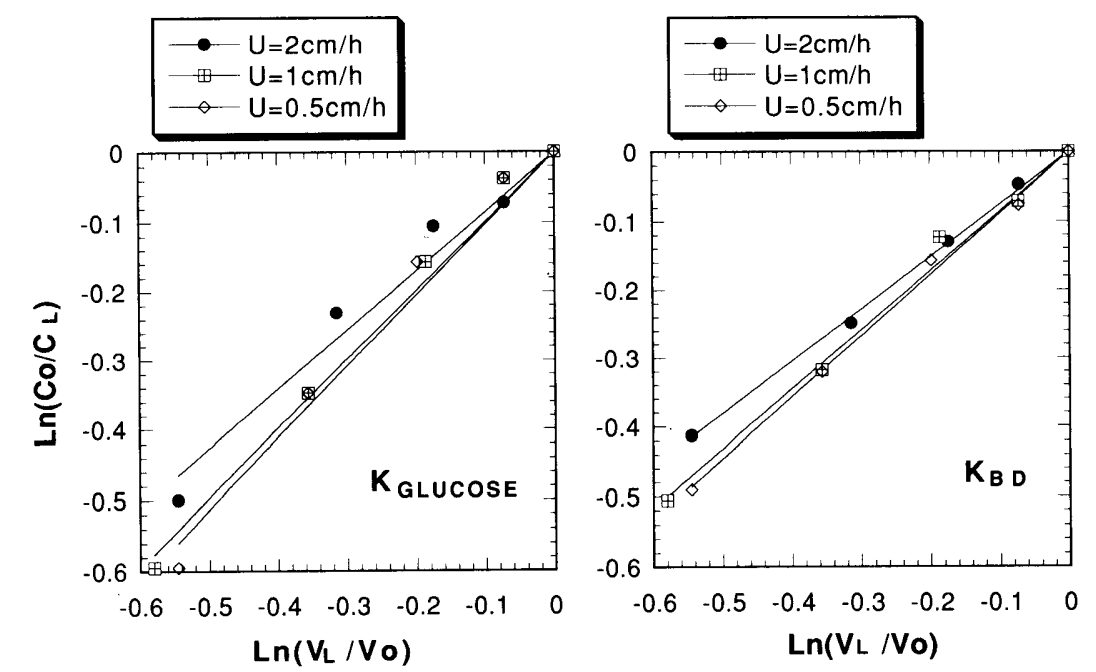

Fig. 6. Determination of apparent partition coefficient of glucose and BD in progressive freeze-concentration of a mixed solution of glucose and BD at a stirring speed of $1400 \mathrm{rpm}$. 


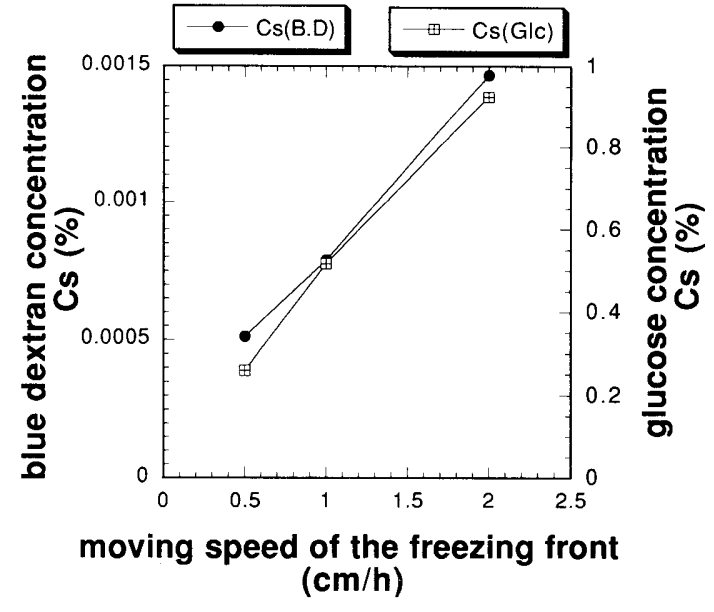

Fig. 7. Effect of moving speed of freezing front on solute concentrations in the ice phase during progressive freeze-concentration of a mixed solution of glucose and BD at a stirring speed of $1400 \mathrm{rpm}$.

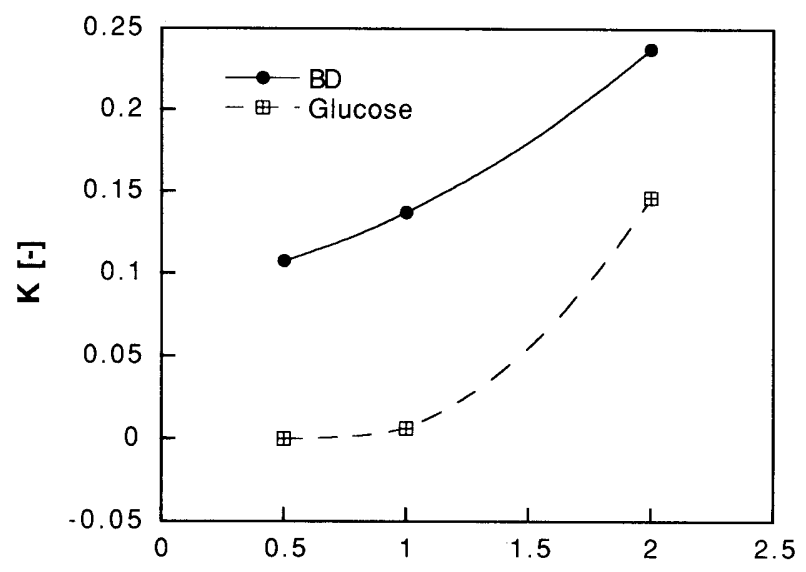

Moving speed of freezing front $(\mathrm{cm} / \mathrm{h})$

Fig. 8. Apparent partition coefficient and its dependence on moving speed of the freezing front in progressive freeze-concentration of a mixed solution of glucose and BD at a stirring speed of $1400 \mathrm{rpm}$.

speed of the ice front resulted in a higher purity in the ice phase. At the moving speed of the ice front of $0.5 \mathrm{~cm} / \mathrm{h}$, the concentration ratio of the solute in the solution and the ice phases was 38 and 24 for glucose and $\mathrm{BD}$, respectively, at $V_{\mathrm{L}} / V_{0}=0.5$.

The apparent partition constant is plotted relative to the moving speed of the freezing front in Fig. 8. Although the absolute values were different between the concentrations of glucose and $\mathrm{BD}$ in the progressive freezing-concentration, the partition constant increased with an increase in the moving speed of the freezing front. The partition constant was lower for glucose compared with BD just as in Fig. 4.

In the progressive freeze-concentration, the major part of the impurity in the ice phase seemed involved in the ice phase when supercooling occurred before the initial crystallization at the bottom of the sample vessel. To suppress the initial supercooling, ice-nucleating protein (Watanabe \& Arai, 1987) was used. As shown in Fig. 9, the ice-nucleating protein

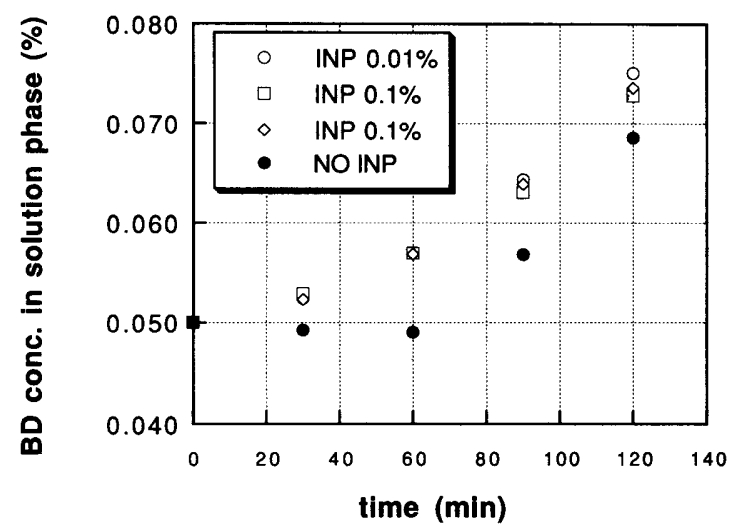

Fig. 9. Effect of ice-nucleating protein (INP) on the freeze-concentration ratio in progressive freeze-concentration of a mixed solution of glucose and $\mathrm{BD}$ (stirring speed, $600 \mathrm{rpm}$; moving speed of the freezing front, $2 \mathrm{~cm} / \mathrm{h}$ ).

was effective in improving the freeze-concentration ratio through the suppression of initial supercooling.

In the conventional method of freeze-concentration, it is most important to have large ice crystals to ease the ice-crystal separation from the concentrated solution phase. Shirai $e t$ al. (1987) proposed a method to utilize ice-crystal agglomeration to grow ice crystals large, and Shimoyamada et al. (1997) pointed out the necessity of the control of supercooling in the crystallizer. Apart from the conventional method, Kobayashi (1991) proposed a unique method of a continuous pressurized freeze-concentration in which ice crystals are pressurized at the outlet to remove the unfrozen concentrated solution effectively.

In the present method of the progressive freeze-concentration, only a single ice crystal grows so that the separation of the ice crystal from the concentrated solution is very easy. At appropriate operating conditions with a high speed stirring at the ice-solution interface and a low speed of ice crystal growth, the concentration efficiency was proved to be high. The progressive freeze-concentration is expected to be applicable to a high quality concentration process of liquid food with a minimal loss of nutrients and flavors and also to a waste water treatment process combined with a low temperature energy recovery system.

Acknowledgments The authors are grateful to Mr. K. Homma, Q.P. Corporation, for the gift of the ice-nucleating protein solution.

\section{References}

Bae, S.K., Miyawaki, O. and Arai, S. (1994). Control of freezing front structure and its effect on the concentration-efficiency in the progressive freeze-concentration. Cryobiol. Cryotechnol, 40, 29-32.

Bayindirli, L., Özilgen, M. and Ungan, S. (1993). Mathematical analysis of freeze concentration of apple juice. J. Food Eng., 19, 95107.

Deshpande, S.S., Bolin, H.R. and Salunkhe, D.K. (1982). Freeze concentration of fruit juices. Food Technol., May, 68-82.

Gouw, T.H. (1968). Normal freezing, In "Progress in Separation and Purification," vol. 1, ed. by E.S. Perry. John Wiley \& Sons, New York, pp. 57-82.

Hartel, R.W. and Espinel, L.A. (1993). Freeze concentration of skim milk. J. Food Eng., 20, 101-120.

Hartel, R.W. and Chung, M.S. (1993). Contact nucleation of ice in fluid dairy products. J. Food Eng., 18, 281-296. 
Huige, N.J.J. and Thijssen, H.A.C. (1972). Production of large crystals by continuous ripening in a stirred tank. J. Cryst. Growth, 13/14, 483-487.

Kobayashi, T. (1991). Freeze concentrations and their application. Reitou, 66, 1008-1018 (in Japanese).

Omran, A.M. and King, C.J. (1974). Kinetics of ice crystallization in sugar solutions and fruit juices. AIChE J., 20, 795-803.

Ramteke, R.S., Singh, N.I., Rekha, M.N. and Eipeson, W.E. (1993). Methods for concentration of fruit juices: a critical evaluation. $J$. Food Sci. Technol., 30, 391-402.

Shimoyamada, M., Shibata, M., Ishikawa, K., Asanuma, K. and Watanabe, K. (1997). Preparation of large ice crystals by controlling the difference between solution and coolant temperatures and its application to freeze concentration. Nippon Shokuhin Kogaku
Kaishi., 44, 59-61 (in Japanese)

Shirai, Y. (1991). Freeze concentration for liquid foods. Kagaku Kogaku, 55, 44-45 (in Japanese).

Shirai, Y., Nakanishi, K., Matsuno, R. and Kamikubo, T. (1985). Effects of polymers on secondary nucleation of ice crystals. J. Food Sci., 50, 401-406.

Shirai, Y., Sugimoto, T., Hashimoto, M., Nakanishi, K. and Matsuno, R. (1987). Mechanism of ice growth in a batch crystallizer with an external cooler for freeze concentration. Agric. Biol. Chem., 51, 2359-2366.

Watanabe, M. and Arai, S. (1987). Freezing of water in the presence of the ice nucleation active bacterium, Erwinia ananas, and its application for efficient freeze-drying of foods. Agric. Biol. Chem., 51, 557-563. 